
\title{
R Reserach S Suare \\ Comparison of Curettage and Hysteroscopy Plus Curettage After Uterine Arterial Embolization in the Treatment of Cesarean Scar Pregnancy
}

\section{Lili Cao}

Women's Hospital, Zhejiang University School of Medicine

\section{Zhida Qian}

Women's Hospital, Zhejiang University School of Medicine

Lili Huang ( $\square$ fbjys@zju.edu.cn )

Women's Hospital, Zhejiang University School of Medicine https://orcid.org/0000-0002-5919-3172

\section{Research article}

Keywords: Cesarean scar pregnancy, Hysteroscopy, Curettage, Uterine artery embolization

Posted Date: July 2nd, 2020

DOI: https://doi.org/10.21203/rs.3.rs-39244/v1

License: (c) (i) This work is licensed under a Creative Commons Attribution 4.0 International License.

Read Full License 


\section{Abstract}

Background: Caesarean scar pregnancy (CSP) stands for the advanced stage severe complication secondary to cesarean section, and its incidence shows an increasing trend recently. However, no consensus has been reached about the optimal CSP treatment.

Methods: The childbearing CSP patients with a cesarean section history were evaluated by ultrasonography, with a gestational age of less than 10 weeks. 34 patients receiving dilation and curettage (D\&C) and uterine artery embolization (UAE) were enrolled into the D\&C group, while 46 undergoing hysteroscopy $(\mathrm{H} / \mathrm{S})$ and $D \& C$ after UAE were enrolled into the H/S+D\&C group.

Results: Differences in success rate and decrease in the $\beta$-hCG level in serum on the second day of surgery were not significant between $D \& C$ and $H / S+D \& C$ groups $(P>0.05)$. Also, differences in side effect rate, intraoperative blood loss amount, postoperative bleeding time, and total length of stay were not significant between both groups $(P>0.05)$. Compared with $D \& C$ group, $H / S+D \& C$ group had decreased postoperative length of stay $(P<0.05)$, increased hospitalization cost $(P<0.05)$, and significantly elevated time of CSP mass disappearance $(P<0.05)$. In addition, 8 (18.19\%) patients in H/S+D\&C group developed anesthesia-related side effects.

Conclusions:This study reveals no obvious difference between UAE+D\&C and H/S+D\&C in terms of the clinical efficacy and safety, except for the time of CSP mass disappearance. The hospitalization cost is more expensive for $\mathrm{H} / \mathrm{S}+\mathrm{D} \& \mathrm{C}$, but the postoperative length of stay is shorter for $\mathrm{H} / \mathrm{S}+\mathrm{D} \& \mathrm{C}$. Besides, $\mathrm{H} / \mathrm{S}+\mathrm{D} \& \mathrm{C}$ is associated with the risk of anesthesia-associated side effects. All these will affect the selection of patients for the two methods.

\section{Background}

Cesarean scar pregnancy (CSP) refers to the pregnancy where the pregnancy sac is implanted at the uterine scar. CSP represents a rare ectopic pregnancy in a special site and a serious long-time complication secondary to cesarean section[6]. Recently, CSP incidence increases significantly worldwide, especially in China. Due to the high rate of cesarean section in China in the past, more and more females are willing to have another baby after cesarean section as the second-child policy is opened up, and the number of CSP cases has increased accordingly [8].

At present, there is no well-recognized optimal treatment for CSP at home and abroad. Typically, the mainstream treatment is to pretreat uterine artery embolization (UAE) and then perform dilation and curettage (D\&C) under ultrasound guidance [1]. However, D\&C can not be performed under direct vision, so the implantation site of the pregnancy sac can not be observed, which results in a risk of uterine perforation and major bleeding by D\&C alone.

In recent years, the removal of CSP pregnancy tissue under hysteroscopy combined with curettage surgery $(H / S+D \& C)$ has been considered as a safe and effective minimally invasive treatment[4]. 
Nonetheless, the H/S + D\&C treatment is associated with certain limitations. For example, doctors should be familiar with the hysteroscopic equipment and be experienced in operation. Meanwhile, hysteroscopy, together with its anesthesia methods, may bring certain complications and cause increased costs, thus potentially increasing the risk and financial burden on patients.

Therefore, to explore the necessity of hysteroscopy after preventive UAE, this study compared the surgical efficacy, safety, costs, and hospital stay of the two treatment methods.

\section{Methods}

\section{Subjects}

CSP was diagnosed according to age, gestational age, medical history, and ultrasound examination of patients. All the enrolled patients were of childbearing age who had previously received cesarean section, with or without menopause. No anatomical abnormality was detected in the reproductive system. All patients did not receive sex hormone therapy, radiation therapy, or chemotherapy within six months before the operation. The gestational age of patients enrolled was less than 10 weeks. The present work enrolled CSP patients who met the above conditions who underwent UAE at the Women's Hospital of Zhejiang University School of Medicine from January 2017 to December 2019. This institution represents the greatest gynecology and obstetrics medical center in Zhejiang, China. A total of 80 CSP cases were enrolled for retrospective analysis. The Women's Hospital of Zhejiang University School of medicine had approved our study protocol (ID:20180018). All patients signed the informed consent to participate in the study.

\section{The diagnosis standard of the gestational type CSP by ultrasonography}

The ultrasonic diagnostic criteria for CSP patients are as follows[7]: $\otimes$ pregnancy sac is not detected within cervical canal or uterine cavity; $₫$ pregnancy sac is seen at scar site on the lower uterus anterior wall, with thinning and interrupted muscle layer; $₫$ color Doppler flow imaging (CDFI) shows high velocity flow signals with low impedance surrounding pregnancy sac, and resistance index (RI) is generally $<0.4$ to 0.5 .

Generally, CSP can be classified as two types[5], namely, gestational sac type and asymmetrical mass type. As for gestational sac type, the gestational sac is embedded into the muscle layer and grows to the bladder or attaches to the scar and grows to the uterine cavity. At the attachment of pregnancy sac, the myometrium may be absent or thin. The asymmetrical mass type is rarer than the former, in which the lesion is mainly mass echo that is solid or cystic in the lower part of the anterior wall.

Also, CSP can be further divided into three types according to the pregnancy sac growth direction and myometrial thickness between the bladder and the fetal sac[3]. In type $\nabla$, partial or most gestational sac is located in uterine cavity, with thinning myometrium lying between bladder and pregnancy sac, and a

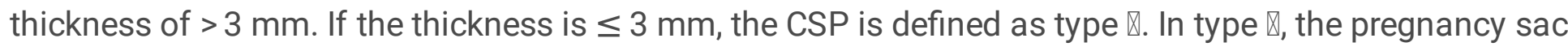


is convex towards the bladder, with significantly thinning or missing myometrium between bladder and

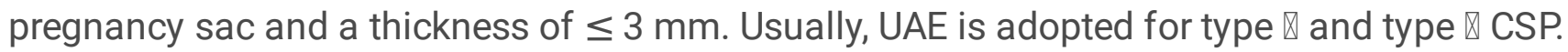

\section{Management}

\section{UAE pretreatment}

All patients received preventive UAE at $24 \mathrm{~h}$ before D\&C or H/S + D\&C. Specifically, the UAE pretreatment was performed by two experienced radiologists under local anesthesia by adopting the super selective "Seldinger" technology, and the bilateral uterine arteries were embolized with gelatin sponge particles. Embolization was confirmed by post-embolization angiography.

\section{D\&C}

34 patients receiving D\&C were enrolled into the $D \& C$ group. All D\&C surgeries were operated by an experienced gynecologist under the assistance of transabdominal ultrasound. First of all, the cervix was carefully expanded. Then, electric negative pressure suction and local curette scraping were applied for removing pregnancy sac along with the local hemorrhage. For reducing risks of major bleeding and uterine perforation, residual pregnancy tissue was removed through scraping gently. Uterine ultrasonography before and after surgery is shown in Fig. 1A and 1C, respectively.

\section{H/s And D\&c}

46 patients undergoing $\mathrm{H} / \mathrm{S}$ and $\mathrm{D} \& \mathrm{C}$ were enrolled into the H/S + D\&C group. All H/S and D\&C surgeries were operated by an experienced gynecologist. Hysteroscopy was conducted to check the implantation site of pregnancy sac, together with uterine cavity condition. Then, under the TAS surveillance, the oval forceps were used to force out the pregnancy sac and its attachment. No prominent residue or local electrocoagulation was applied in treating bleeding points. After satisfactory inspection, this operation was completed. Uterine ultrasonography before and after surgery is presented in Fig. 1B and 1D, respectively.

\section{Research Contents}

For the two groups of patients who were collected before surgery, their clinical features were extracted, including maternal age, gravidity time, abortion time, the time duration from CS to CSP, diameter of pregnancy sac under ultrasound, gestational age,, thickness of cesarean scar, preoperative $\beta$-hCG level in serum, prior CS number, fetal cardiac activity, vaginal bleeding, and CSP types. Clinical data were collected from these two groups of patients after surgery, including 4 indicators of efficacy (the declined blood $\beta$-HCG level on the first day following operation, bleeding time, $\beta$-hCG content in serum as well as mass disappearance of CSP after surgery), 3 indicators of safety (successful rate of osurgery, 
intraoperative blood loss amount the surgery-related complications), 3 indicators of cost-effectiveness ratio (total length of hospital stay, postoperative length of stay, and hospitalization costs) and anesthesia-related side effects. In this study, the complications were hemorrhagic shock and infection. All patients were followed up after surgery. The blood $\beta$-HCG level was reviewed every week till it restored to physiological level (<5.3 IU/L). In addition, transvaginal ultrasound was reviewed at $0.1,1,2$ and 3 months postoperatively, so as to dissect local residues of uterine scars until they completely disappeared.

\section{Statistical Methods}

SPSS 20.0 was employed for data analysis. P-value indicated the two-sided probability and a difference of $P<0.05$ indicated statistical significance. First of all, all patients were subjected to the KolmogorovSmirnov test (normal distribution test) together with the variance homogeneity test (Levene's Test). For normally distributed data with homogenous variance, the independent sample t-test was used, while for abnormally distributed data with heterogeneous variance, the Mann-Whitney UTest (non-parametric test) was adopted. Meanwhile, data regarding the composition ratio were measured by the chi-square test $\left(\chi^{2}\right.$ Test).

\section{Results}

\section{General information}

There were no statistical differences in maternal age, gravidity, abortion times, the duration from CS to CSP, diameter of pregnancy sac, gestational age, preoperative $\beta$-hCG level in serum, prior CS number, cases with fetal cardiac activity, cases having preoperative vaginal bleeding or cases with different CSP types between D\&C and H/S + D\&C groups. (Table 1) 
Table 1

Characteristics of patients in D\&C group and H/S + D\&C group

\begin{tabular}{|llll|}
\hline Characteristics & D\&C group & H/S + D\&C group & $\begin{array}{l}\text { P } \\
\text { value }\end{array}$ \\
\hline Maternal age & $35.12 \pm 3.52$ & $33.89 \pm 3.84$ & 0.148 \\
\hline Gravity (times) & $3.71 \pm 1.47$ & $3.26 \pm 1.10$ & 0.126 \\
\hline Abortion (times) & $2.26 \pm 1.31$ & $1.87 \pm 0.91$ & 0.115 \\
\hline Interval between CS and CSP (months) & $83.26 \pm 45.86$ & $80.20 \pm 39.17$ & 0.748 \\
\hline Gestational age (days) & $54.88 \pm 7.96$ & $54.11 \pm 8.73$ & 0.685 \\
\hline Gestational sac diameter (cm) & $3.90 \pm 1.36$ & $3.46 \pm 1.39$ & 0.168 \\
\hline Thickness of cesarean scar (mm) & $1.29 \pm 1.27$ & $1.63 \pm 1.69$ & 0.318 \\
\hline Serum $\beta$-hCG level before surgery (IU/L) & $82027.38 \pm$ & $75754.09 \pm$ & 0.638 \\
\hline Number of prior CS (Times) & 48901.28 & 64865.42 & 0.934 \\
\hline Cases with fetal cardiac activity [n (\%)] & $1(1-2)$ & $1(1-2)$ & 0.494 \\
\hline Cases with vaginal bleeding before surgery [n & $26(76.47)$ & $32(69.57)$ & 0.555 \\
\hline (\%)] & & $24(52.17)$ & 0.949 \\
\hline CSP types & & & $39(84.78)$ \\
\hline II [n (\%)] & $29(85.29)$ & $7(15.22)$ & \\
\hline III [n (\%)] & $5(14.71)$ & & \\
\hline
\end{tabular}

\section{Comparison Of Efficacy Indicators}

The clinical outcome data are displayed in Table 2. Clearly, there were no statistically significant differences in the reduced $\beta$-hCG level in serum on the second day following operation $(76.05 \pm 10.65$ vs. $78.58 \pm 9.52 \%, P=0.268)$, bleeding time [7.5(3-37) vs. $6(3-21), P=0.547]$ or serum $\beta$-hCG resolution $[30(20-71)$ vs. 32(22-74), $P=0.329]$ between $D \& C$ and $H / S+D \& C$ groups. 
Table 2

Comparison outcomes of patients in D\&C group and H/S + D\&C group

\begin{tabular}{|llll|}
\hline Characteristics & D\&C group & H/S + D\&C group & $\begin{array}{l}\text { P } \\
\text { value }\end{array}$ \\
\hline Success rate [n (\%)] & $33(97.06)$ & $46(100)$ & 0.242 \\
\hline Decline of serum $\beta$-hCG the day after surgery (\%) & $76.05 \pm 10.65$ & $78.58 \pm 9.52$ & 0.268 \\
\hline Total hospitalization times (days) & $5(3-12)$ & $5(4-8)$ & 0.472 \\
\hline Hospitalization time after surgery(days) & $3(2-7)$ & $2(2-4)$ & 0.047 \\
\hline Estimated intraoperative blood loss [mL) & $15(5-600)$ & $20(5-250)$ & 0.298 \\
\hline Hospitalization cost (CNY) & $13002.06 \pm$ & $14974.83 \pm$ & 0.000 \\
\hline Side effect rate [n (\%)] & 613.10 & 827.09 & 0.096 \\
\hline Time of bleding after surgery (days) & $2(5.88)$ & $0(0)$ & 0.547 \\
\hline $\begin{array}{l}\text { Time of serum } \beta \text {-hCG resolution after surgery } \\
\text { (days) }\end{array}$ & $7.5(3-37)$ & $6(3-21)$ & 0.329 \\
\hline Time of CSP mass disappearance (days) & $30(20-71)$ & $32(22-74)$ & 0.005 \\
\hline Anesthesia-related side effects [n(\%)] & - & $14(0-126)$ & \\
\hline
\end{tabular}

Compared with D\&C group, the time of CSP mass disappearance was significantly shorter in H/S + D\&C group [37(0-132) vs. $14(0-126)$ days, $P=0.005]$.

\section{Comparison Of Safety Indicators}

Difference in success rate was not statistically significant between D\&C and H/S + D\&C groups (97.06\% vs. $100 \%, P=0.242)$. Among the 80 patients enrolled, 79 patients were treated successfully, while 1 in D\&C group failed in the treatment. This patient developed vaginal bleeding with infection for 23 days after D\&C surgery. On the 23rd day after D\&C, the patient suffered from hemorrhagic shock due to massive vaginal bleeding, so she was admitted again for D\&C surgery with UAE pretreatment. Hysteroscopy was performed due to the poor surgical results of D\&C. Eventually, the blood loss amount in this patient was significantly reduced, and villous tissue was detected in the blood clot removed from the scar.

Differences in incidence of operation-related side effects [2(5.88) vs. $0(0), P=0.096$ ], and intraoperative blood loss amount [15(5-600) vs. 20(5-250), $P=0.298]$ between $D \& C$ and H/S + D\&C groups were not statistically significant. 
Difference in incidence of side effects showed no statistical significance between D\&C and H/S + D\&C groups [2(5.88\%) vs 0(0), $\mathrm{P}=0.096$ ]. 2 patients in $\mathrm{D} \& \mathrm{C}$ group developed a pelvic infection, of them, one was the failed case, while the other one was discovered with pelvic infection at 7 days after surgery. The patient suffered from vaginal bleeding at 17 days preoperatively and was discharged at 3 days after surgery. All patients in H/S + D\&C group had no side effects (pelvic infection or DIC). During the H/S + D\&C surgery, 15 patients had intrauterine adhesions and were treated with operative hysteroscopy.

\section{Comparison Of Cost-effectiveness Ratio Indicators}

Compared with D\&C group, H/S + D\&C group had significantly decreased postoperative length of stay $[3(2-7)$ vs. $2(2-4)$ days, $P=0.047]$ and remarkably increased hospitalization cost $(13002.06 \pm 613.10$ vs $14974.83 \pm 827.09$ CNY, $P=0.000$ ).

Difference in total length of hospital stay was not statistically significant between $D \& C$ and H/S + D\&C groups [5(3-12) vs. 5(4-8), $P=0.472]$.

\section{Anesthesia-related Side Effects In H/s + d\&c Group}

8 patients in H/S + D\&C group suffered from the anesthesia-related side effects. Among them, one had headache, and no treatment was performed except for emotional comfort. The headache in this patient disappeared one the day after surgery. In addition, 7 of these patients had urinary retention and all of them recovered after catheterization and were discharged. Besides, 1 of the 7 patients with urinary retention was positive for occult blood due to catheter friction.

\section{Discussion}

This study found that, differences in the success rate and decreased $\beta$-hCG level in serum on the first day following operation were not significant between D\&C and H/S + D\&C groups. Such differences may be caused by incomplete removal of pregnancy tissues or residual trophoblasts, no matter which treatment is adopted. Therefore, regularly monitoring the changes in blood $\beta$-hCG level after surgery is of great significance to judge the effect of surgical treatment. In the present work, postoperative $\beta$-hCG resolution time in serum of D\&C and H/S + D\&C groups was 30 (20-71) and 32 (22-74), respectively, which seemed to be longer than that reported in previous studies[2]. For all the studies mentioned in that article, the serum $\beta$-hCG resolution time was less than one month. One possible reason for this phenomenon is that different surgical methods are used. To prevent uterine rupture, doctors just remove the pregnancy tissue as much as possible, which may not be the same as transvaginal hysterotomy.

Also, differences in the incidence of operation-related side effects, intraoperative blood loss amount, postoperative bleeding time, and total length of hospital stay were not significant. However, compared with UAE plus D\&C group, UAE combined with H/S and D\&C group had decreased postoperative length of 
stay, increased hospitalization cost, and significantly decreased time of CSP mass disappearance. Generally speaking, the length of hospital stay and the hospitalization cost will partially affect the patient's choice of treatment. Although the time of CSP mass disappearance was different in the two groups, their time of serum $\beta$-hCG resolution was similar, indicating a high possibility that the existing mass was the parent tissue.

One patient in UAE combined with D\&C group failed. This may be related to the blindness of D\&C surgery, which can not be performed directly and villi tissue can not be cleaned up in time. 15 patients in $\mathrm{H} / \mathrm{S}+$ D\&C group were found to develop intrauterine adhesions, and they were treated with operative hysteroscopy. For patients with the demands for pregnancy, they avoided a second operation for intrauterine adhesions.

This study indicated that, anesthesia brought additional side effects to the patients. Generally, urinary retention is the most likely side effect of anesthesia, which can be completely relieved after catheterization. However, the insertion of a urinary catheter may also cause damage to the ureter. These anesthesia-related side effects are not serious, but they may affect the patient's choice of surgical method. Some patients who lack the anesthesia knowledge may not know which anesthesia method to choose. In addition, patients have a deep fear of the possible anesthesia-associated side effects, in this regard, they may prefer the D\&C surgery. Also, patients may have no idea of symptom severity and are worried about the use of anesthesia. In this case, the surgeon and anesthetist should communicate with the patients before the operation, so as to ease their nervousness. Noteworthily, the precise use of anesthetic drugs can effectively reduce the risk of anesthesia-related side effects and the doctors can well manage the possible complications.

However, certain shortcomings should be noted in this study. Firstly, in this study, all patients were pretreated with UAE. Due to the lack of control, it remains to be further investigated about whether UAE plays an important role, and whether it is sufficient to affect the use of hysteroscopy. Secondly, there were only less than 10 cases with mass CSP from January 2017 to December 2019, and most of them had received hysteroscopy previously; as a result, it was not analyzed in this study.

\section{Conclusions}

Both D\&C and H/S + D\&C after UAE can successfully terminate the gestational type CSP. Apparently, hysteroscopy displays significant advantages in terms of the time of CSP mass disappearance and the postoperative length of stay. Nonetheless, the application of hysteroscopy results in higher hospitalization cost and brings additional anesthesia-related risks. Therefore, treatment should be individualized based on the patient characteristics, meanwhile, the patient feelings should be considered, and their wishes should be fully respected. Also, the medical conditions of each hospital and the technical skills of doctors are different, which should be considered when selecting an appropriate surgical method. According to this study, UAE followed by D\&C is more suitable for patients with the gestational age of less than 70 days who are worried about anesthesia and high cost. Hysteroscopy is 
more appropriate for patients who are highly suspected of intrauterine adhesions before surgery and are willing to get pregnant again.

\section{Declarations}

\section{Declarations}

Ethics approval and consent to participate: The Women's Hospital of Zhejiang University School of medicine had approved our study protocol (ID:20180018). All patients signed the informed consent to participate in the study.

Consent for publication: Not applicable.

Availability of data and materials: All data generated or analysed during this study are included in this published article.

Competing interests: The authors declare that they have no competing interests.

Funding: The present study was funded by the Program of Zhejiang Province Medical and Health Technology Projects. (No. 2018KY430).

Authors' contributions: Lili Cao has made contributions to the acquisition, analysis and interpretation of data. Zhida Qian has made contributions to the design of the work. Lili Huang has made contributions to the conception

Acknowledgements: Thanks to the colleagues in the obstetrics and gynecology department for their support and help when we collect data.

\section{References}

1. Chen H, Zhou J, Wang H, Tan W, Yao M, Wang X. The Treatment of Cesarean Scar Pregnancy with Uterine Artery Embolization and Curettage as Compared to Transvaginal Hysterotomy. Eur J Obstet Gynecol Reprod Biol. 2017;214:44-9.

2. Chen YQ, Liu HS, Li WX, Deng C, Hu XW, Kuang PJ. Efficacy of transvaginal debridement and repair surgery for cesarean scar pregnancy: a cohort study compared with uterine artery embolism. INT J CLIN EXP MED. 2015;8:21187-93.

3. Chen ZY, Li XY, Zhao D, Zhou M, Xu P, Huang XF, Zhang XM. 2017 [Clinical analysis on hysteroscopic surgery for the treatment of type cesarean scar pregnancy in the first trimester] Zhonghua Fu Chan Ke Za Zhi 52 669-74.

4. Int J Gynaecol Obstet 135158 - 62.

5. Qian ZD, Huang LL, Zhu XM. Curettage or operative hysteroscopy in the treatment of cesarean scar pregnancy. ARCH GYNECOL OBSTET. 2015;292:1055-61. 
6. Rotas MA, Haberman S, Levgur M. Cesarean scar ectopic pregnancies: etiology, diagnosis, and management. OBSTET GYNECOL. 2006;107:1373-81.

7. Shih JC. Cesarean scar pregnancy: diagnosis with three-dimensional (3D) ultrasound and 3D power Doppler. Ultrasound Obstet Gynecol. 2004;23:306-7.

8. Wei LK, Yu LM, Mu RM, Xue FX. [Reproductive outcomes following women with previous cesarean scar pregnancy]. Zhonghua Yi Xue Za Zhi. 2018;98:2194-7.

\section{Figures}

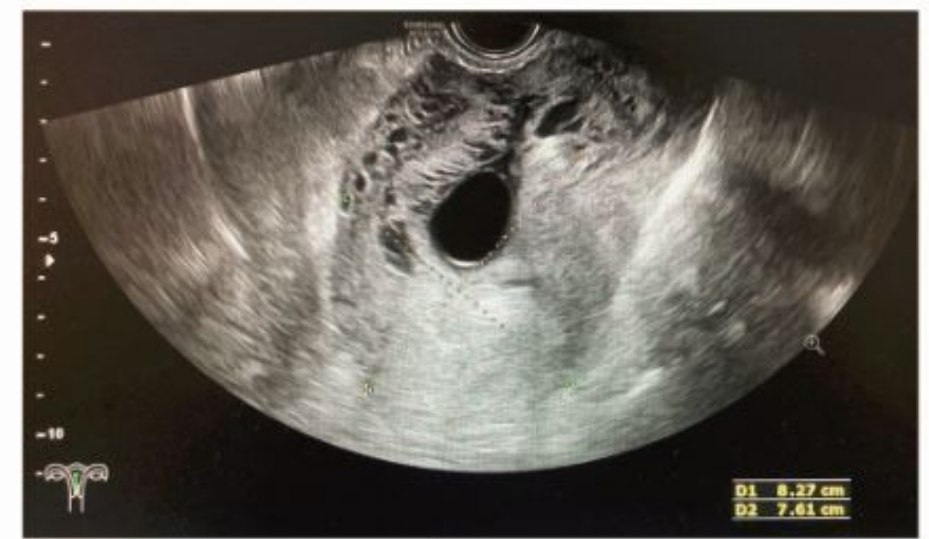

A

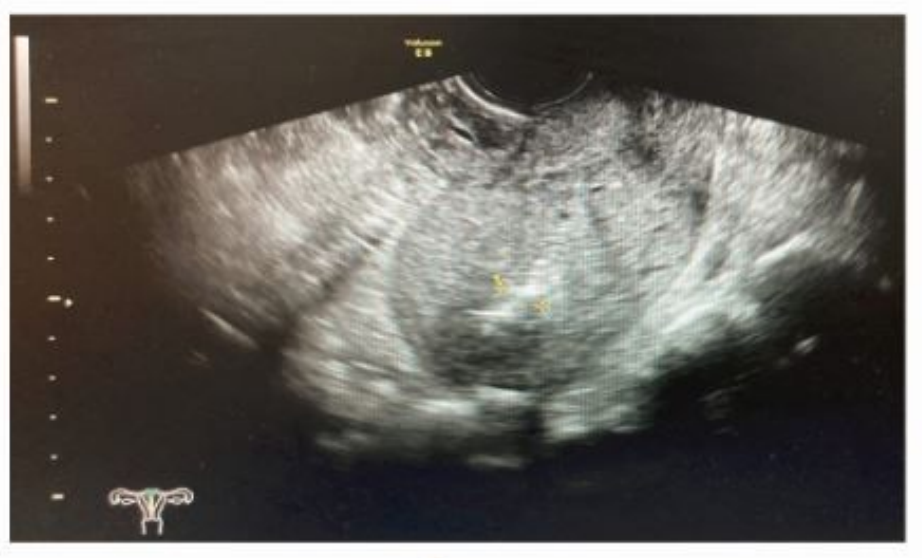

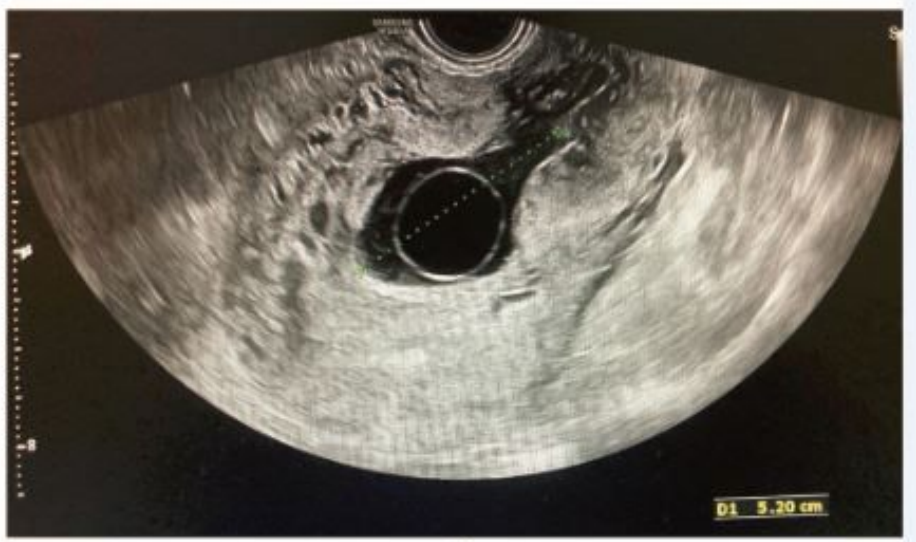

B

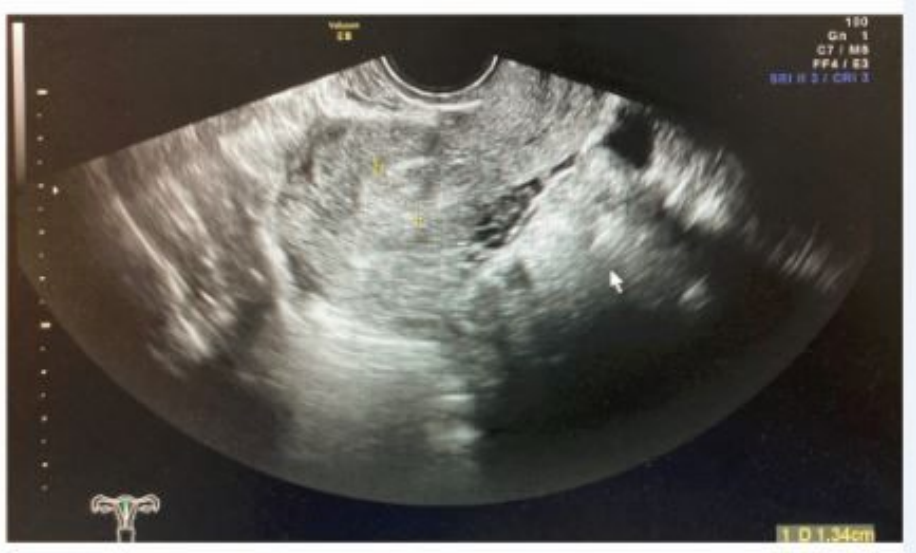

\section{Figure 1}

CSP ultrasonography. A. Transvaginal ultrasonography revealing CSP before D\&C. B. Transvaginal ultrasonography showing CSP before H/S+D\&C. C. Transvaginal ultrasonography showing CSP after D\&C. D. Transvaginal ultrasonography showing CSP after H/S+D\&C. 